\title{
Identificação do consumo de substâncias psicoativas entre indivíduos com esquizofrenia*
}

\author{
Mayara Rodrigues Contin ${ }^{1}$ \\ Clarissa Mendonça Corradi-Webster ${ }^{1}$ \\ Fernanda de Sousa Vieira ${ }^{1}$ \\ Ana Carolina Guidorizzi Zanetti ${ }^{2}$
}

\begin{abstract}
Este estudo teve por objetivo identificar o consumo problemático de substâncias em uma amostra de 45 pessoas diagnosticadas com esquizofrenia, tratadas em Centro de Atenção Psicossocial II, e verificar a associação desse consumo a características sociodemográficas e clínicas dos participantes. Estudo transversal. Foram aplicados questionários sobre as substâncias mais consumidas ao longo da vida: tabaco (67\%), álcool (73\%) e maconha (22\%). Dos participantes, $62 \%$ faziam uso problemático de pelo menos uma droga. Encontrou-se associação entre uso problemático de substâncias e satisfação financeira, impulsividade e experiências de discriminação. Conhecer as características relacionadas ao uso de drogas entre pessoas em tratamento para esquizofrenia auxilia a propor estratégias de cuidado para essa população.
\end{abstract}

Descritores: Comorbidade; Esquizofrenia; Transtornos Relacionados ao Uso de Substâncias.

\footnotetext{
* Apoio financeiro da Fundação de Amparo à Pesquisa do Estado de São Paulo (FAPESP), Brasil, processo n 2011/22739-2.

${ }^{1}$ Universidade de São Paulo, Faculdade de Filosofia, Ciências e Letras de Ribeirão Preto, Ribeirão Preto, SP, Brasil.

${ }^{2}$ Universidade de São Paulo, Escola de Enfermagem de Ribeirão Preto, Centro Colaborador da OPAS/OMS para o Desenvolvimento da Pesquisa em Enfermagem, Ribeirão Preto, SP, Brasil.
} 


\title{
Identification of psychoactive substance use among individuals diagnosed with schizophrenia
}

The objective was to identify the consumption of substances among people diagnosed with schizophrenia undergoing treatment and to verify its association with sociodemographic and clinical characteristics. Questionnaires were applied in 45 patients. Substances most commonly consumed throughout life: $67 \%(n=30)$ tobacco; $73 \%(n=33)$ alcohol; $22 \%(n=10)$ marijuana. $62 \%$ of the sample made problematic use of at least one drug. There was an association between problematic drug use and financial satisfaction, impulsiveness and experiences of discrimination. Problematic use of tobacco was associated with street living. Knowing characteristics related to the use of drugs among people in treatment for schizophrenia helps to propose strategies of care directed to this population.

Descriptors: Comorbidity; Schizophrenia; Substance-Related Disorders.

\section{La identificación del consumo de sustancias entre los individuos con diagnóstico de esquizofrenia}

\begin{abstract}
El objetivo fue identificar el uso de sustancias entre las personas diagnosticadas con esquizofrenia y verificar la asociación entre el consumo y las características sociodemográficas y clínicas. Estudio transversal. Se utilizaron cuestionarios en 45 pacientes con diagnóstico de esquizofrenia. Las sustancias de mayor consumo en la vida: $67 \%$ tabaco; $73 \%$ alcohol; $22 \%$ marihuana. $62 \%$ de la muestra hicieron uso problemático de al menos una sustancia. Se encontró una asociación entre el uso problemático de drogas y la satisfacción financiera, la impulsividad y las experiencias de discriminación. Identificar las características relacionadas con el consumo de drogas entre personas que reciben tratamiento para la esquizofrenia ayuda a propuesta de estrategias de atención dirigida a esta población.
\end{abstract}

Descriptores: Comorbilidad; Esquizofrenia; Trastornos Relacionados con Sustancias.

\section{Introdução}

Nos últimos 20 anos, o consumo de drogas entre pessoas diagnosticadas com transtornos mentais tem sido foco de investigações clínicas ${ }^{(1-2)}$. Entre esses estudos, destacam-se aqueles envolvendo a população com diagnóstico de esquizofrenia e outros transtornos psicóticos $^{(3)}$.

Estudo realizado nos Estados Unidos sobre diagnóstico duplo na população geral mostrou que, entre os participantes diagnosticados com esquizofrenia, $47 \%$ preenchia os critérios para algum tipo de consumo problemático de álcool ou outras drogas ${ }^{(4)}$. Outro estudo conduzido com pessoas diagnosticadas com esquizofrenia encontrou que $54 \%$ dos participantes apresentavam diagnóstico de problemas relacionados ao álcool e outras drogas ${ }^{(5)}$. No Brasil, estudo realizado com pacientes psiquiátricos mostrou que, entre os participantes diagnosticados com esquizofrenia, $8 \%$ deles apresentavam uso problemático de drogas, dos quais, 5,3\% tinham problemas com o uso de álcool e $2,7 \%$ com outras drogas $^{(6)}$. Outro estudo nacional encontrou que, das pessoas com diagnóstico de esquizofrenia, as que faziam uso problemático de drogas eram significativamente mais jovens do que as pessoas que não apresentavam consumo de drogas ${ }^{(7)}$. 
Os autores não encontraram diferenças significativas quanto ao gênero e tempo de hospitalização.

Embora a proporção de pessoas que consomem drogas seja superior entre aquelas com diagnóstico de esquizofrenia, a literatura ressalta que os números absolutos são pequenos(8). São variados os tipos de drogas consumidas, bem como os padrões de consumo nessa população. Destacam-se o álcool, o tabaco e a maconha, que apresentam estatísticas de consumo variadas nos estudos, de acordo com aspectos culturais e métodos de pesquisa empregados ${ }^{(6,9)}$.

É importante enfatizar que uma das possíveis explicações para o uso de drogas entre pessoas com esquizofrenia é o alívio que pode proporcionar em relação aos sintomas depressivos e ansiosos, no alcance ou na manutenção da euforia, como estratégia de automedicação, melhorando a autoconfiança e as habilidades sociais ${ }^{(10)}$. Outra questão a ser considerada é que as drogas têm sido utilizadas em todas as culturas conhecidas, sendo um recurso utilizado pela população de modo geral. Ressalta-se que o consumo de álcool é um hábito comum, talvez mais legitimado socialmente se comparado a outras substâncias, e pode estar relacionado a um sentimento de normalidade ${ }^{(10)}$. Por isso, entre pacientes com diagnóstico de esquizofrenia, o álcool pode ser usado como estratégia de socialização ou como tática para esquecer situações traumáticas, ao menos nos períodos em que as pessoas estão sob seu efeito(11).

O consumo de cigarro, por sua vez, parece ser uma prática comum em ambientes psiquiátricos, assumindo características rituais e de hábitos cotidianos. O cigarro se relaciona ao alívio de estresse, tomando um sentido de controle da própria vida para pessoas com grande experiência de institucionalização(12). Embora o tabaco seja reportado como uma substância sem influência na saúde mental de pessoas com diagnósticos psiquiátricos, estudo recente relacionou o consumo de cigarro a experiências psicóticas. O tabaco foi descrito como uma variável que intervém nos resultados, quando relacionado ao consumo de maconha, amplamente estudado(13-16). A maconha, em estudos qualitativos, tem sido relacionada à sensação de perspectiva de energia e criatividade, em pessoas sob efeito da droga, mas também se relaciona à diminuição das alucinações auditivas ${ }^{(17-18)}$.

Embora muitos usuários percebam alguns benefícios com o uso de substâncias, esse consumo pode exacerbar sintomas negativos e positivos, resultando em um conjunto de danos cognitivos e nas funções afetivas, biológicas e interpessoais ${ }^{(10,19)}$. Assim, discute-se que esses indivíduos podem apresentar um pior prognóstico quando comparados a pessoas que possuem apenas um dos transtornos, dificultando o tratamento(20). Nos serviços de saúde mental, é importante identificar precocemente os sintomas que preenchem os critérios diagnósticos para esquizofrenia entre as pessoas que buscam tratamento e que fazem uso problemático de substâncias. O cuidado integrado ao indivíduo pode ter efeitos benéficos para o tratamento e evolução do transtorno.

Frente a isso, este estudo teve como objetivo identificar o consumo de drogas entre pessoas com diagnóstico de esquizofrenia, verificando sua associação com características sociodemográficas e clínicas.

\section{Método}

Estudo quantitativo, descritivo e transversal realizado em um Centro de Atenção Psicossocial II (CAPS II) de um município do interior paulista. A amostra de conveniência foi composta por 45 pacientes que preencheram os seguintes critérios de inclusão: estar em seguimento ativo no serviço selecionado; ter diagnóstico de esquizofrenia; ter mais que 18 anos de idade.

Para coleta de dados, foram utilizados três instrumentos: 1) formulário de informações sociodemográficas e clínicas (sexo, idade, estado civil, religião, situação ocupacional e educacional, renda familiar, com quem reside, satisfação com a comunidade onde reside, filhos menores de 18 anos, problemas com álcool e outras substâncias na família, diagnóstico psiquiátrico, internações psiquiátricas, uso de medicamentos, experiência de vivência na rua, problemas legais e situações de discriminação); 2) Questionário para Triagem do Uso de Álcool, Tabaco e outras Substâncias (ASSIST) (21-22); e 3) Barratt Impulsiveness Scale (BIS-11)(23).

O ASSIST foi desenvolvido pela Organização Mundial da Saúde. Trata-se de um questionário estruturado, composto por oito questões que investigam o consumo de nove substâncias (tabaco, álcool, maconha, cocaína, estimulantes, sedativos, inalantes, alucinógenos e opiáceos) $)^{(21-22)}$. As questões rastreiam o consumo de substâncias psicoativas, identificando seu uso ao longo da vida, uso nos últimos três meses, problemas relacionados ao consumo, risco de danos, dependência e uso injetável. Cada resposta corresponde a um escore, que varia de zero a quatro, e a soma total pode variar de zero a 20. Escores entre zero e três indicam uso de baixo risco, entre quatro e 15 , uso abusivo, e acima de 16, possível dependência. No teste de avaliação da consistência interna da versão em português do instrumento, o valor do a de Cronbach encontrado foi de 0,8 para álcool e tabaco, 0,79 para maconha e 0,81 para cocaína ${ }^{(21)}$. 
A BIS-11 foi utilizada para avaliar a impulsividade. Esse instrumento foi adaptado para uso entre adultos no Brasil( $^{(23)}$. É uma escala de autorrelato, composta por 30 itens que descrevem manifestações de impulsividade, cuja pontuação varia entre 30 e 120, adotando-se o índice de 72 pontos para classificar a impulsividade como baixa ou elevada ${ }^{(24)}$.

Os pacientes foram contatados no CAPS II pelos membros da equipe de pesquisa e convidados a participar. Os instrumentos foram aplicados em uma sala disponibilizada pelo serviço e o tempo para coleta variou entre 30 e 45 minutos. Cabe destacar que todas as informações foram coletadas durante a entrevista com o paciente, exceto o diagnóstico, retirado das fichas médicas que continham as informações referentes à consulta do dia.

Os dados foram organizados em uma planilha do programa Excel e submetidos a análise estatística. As análises descritivas e inferenciais foram feitas com o Statistical Package for Social Sciences (SPSS), versão 16. Para as variáveis categóricas, foram calculadas as frequências e seus respectivos percentuais. Para análise inferencial foi utilizado o teste Exato de Fisher. Os participantes foram classificados em dois grupos. As pessoas que pontuaram positivamente para o uso problemático de pelo menos uma das drogas avaliadas pelo ASSIST $(n=9)$ foram colocadas no grupo Uso Problemático de Drogas e as demais, no grupo Sem Uso Problemático de Drogas. Destacase que no grupo "com Uso Problemático de Drogas" não foram colocados aqueles que pontuaram positivo apenas para o tabaco, substância com características farmacológicas e culturais que, apesar de causar dependência e consequências clínicas diversas, tem pouca influência no funcionamento psicossocial dos indivíduos. Pesquisou-se a associação entre esses dois grupos e as variáveis sociodemográficas e clínicas. O nível de significância adotado foi de $5 \%$ ( $p \leq 0,05)$.

O projeto foi aprovado pelo Comitê de Ética em Pesquisa da Faculdade de Filosofia, Ciências e Letras de Ribeirão Preto da Universidade de São Paulo (FFCLRP-USP) em 30 de maio de 2012 (CAAE 00670512.9.0000.5407).

\section{Resultados}

Os 45 participantes referiram maiores médias para o consumo de tabaco $(10,1, d p=12)$ e álcool $(4,1, d p=8)$. Em relação ao padrão de consumo, com exceção dos opioides, os participantes referiram ter feito uso de todas as outras substâncias pelo menos uma vez na vida. Quanto ao uso abusivo, foi mais prevalente para o consumo de tabaco (36\%), seguido do álcool (7\%) e da maconha (7\%). No que tange à dependência, 13\% estavam relacionadas ao tabaco; $7 \%$, ao álcool; e 2\%, a anfetaminas e ecstasy (Tabela 1). Se incluído o tabaco, 28 pessoas $(62,2 \%)$ faziam uso problemático de pelo menos uma substância.

Em relação às características sociodemográficas dos participantes, a maioria era do sexo masculino (53\%), não era casada ou apresentava relacionamento estável $(82 \%)$, residia com familiares $(75 \%)$, não trabalhava (84\%), não tinha renda própria (71\%) e não estava satisfeita com sua situação financeira (60\%). Para o teste de associação entre o uso de drogas e as características sociodemográficas e clínicas, os participantes foram divididos entre aqueles que faziam consumo problemático de pelo menos uma droga ( $n=9,2 \%$ ) e os que não faziam. Encontrou-se associação significativa apenas entre o uso problemático de drogas e a insatisfação com a situação financeira ( $p=0,016)$, conforme Tabela 2 .

Tabela 1: Média de pontuação no ASSIST para cada uma das drogas avaliadas e frequência e porcentagem de diferentes padrões de consumo $(n=45)$

\begin{tabular}{|c|c|c|c|c|c|c|c|}
\hline \multirow[b]{2}{*}{ Substância } & \multirow{2}{*}{$\begin{array}{l}\text { Pontuação } \\
\text { M (dp) }\end{array}$} & \multicolumn{2}{|c|}{ Uso na Vida } & \multicolumn{2}{|c|}{ Uso abusivo } & \multicolumn{2}{|c|}{ Provável dependência } \\
\hline & & $\begin{array}{c}\text { Sim } \\
N(\%)\end{array}$ & $\begin{array}{c}\text { Não } \\
\text { N (\%) }\end{array}$ & $\begin{array}{c}\text { Sim } \\
N(\%) \\
\end{array}$ & $\begin{array}{c}\text { Não } \\
\text { N (\%) }\end{array}$ & $\begin{array}{c}\text { Sim } \\
N(\%)\end{array}$ & $\begin{array}{c}\text { Não } \\
N(\%)\end{array}$ \\
\hline Tabaco & $10,1(12)$ & $30(67)$ & $15(33)$ & $16(36)$ & $29(64)$ & $6(13)$ & $39(88)$ \\
\hline Álcool & $4,1(8)$ & $33(73)$ & $12(27)$ & $3(7)$ & $42(93)$ & $3(7)$ & $42(93)$ \\
\hline Maconha & $0,7(3)$ & $10(22)$ & $35(78)$ & $3(7)$ & $42(93)$ & 0 & $45(100)$ \\
\hline Cocaína/crack & $0,2(1)$ & $4(9)$ & $41(91)$ & $1(2)$ & $44(98)$ & 0 & $45(100)$ \\
\hline Anfetaminas/ecstasy & $0,8(6)$ & $2(4)$ & $43(96)$ & 0 & $45(100)$ & $1(2)$ & $44(98)$ \\
\hline Inalantes & $0(0)$ & $3(7)$ & $42(93)$ & 0 & 0 & 0 & $45(100)$ \\
\hline Alucinógenos & $0,1(1)$ & $1(2)$ & $44(98)$ & $1(2)$ & $44(98)$ & 0 & $45(100)$ \\
\hline Opioides & $0(0)$ & $0(0,0)$ & $45(100)$ & 0 & $45(100)$ & 0 & $45(100)$ \\
\hline Uso de pelo menos uma droga & - & $38(84)$ & $7(16)$ & $19(42)$ & $26(58)$ & $9(20)$ & $36(80)$ \\
\hline
\end{tabular}


A maioria dos participantes relataram ter algum familiar que fazia uso problemático de drogas (51\%), não ter dificuldades em tomar os medicamentos prescritos (73\%), ter experiência de hospitalização psiquiátrica (80\%), ter alta impulsividade $(52 \%)$, não ter experiência de ter sido violentado (53\%), discriminado (57\%), ter vivido nas ruas (84\%) ou ter histórico de problemas com a polícia $(78 \%)$. Conforme ilustrado na Tabela 3, houve associação significativa entre uso problemático de drogas e impulsividade $(p=0,02)$ e experiência de discriminação $(p=0,003)$.

Tabela 2: Características sociodemográficas dos pacientes psiquiátricos, de acordo com a presença ou ausência de uso problemático de drogas

\begin{tabular}{|c|c|c|c|c|c|}
\hline \multirow[b]{2}{*}{ Variável } & \multirow[b]{2}{*}{ N válido } & \multicolumn{2}{|c|}{ Uso problemático de drogas } & \multirow[b]{2}{*}{ P-value } & \multirow{2}{*}{$\begin{array}{c}\text { OR } \\
95 \% \text { IC }\end{array}$} \\
\hline & & $\begin{array}{c}\text { Não } \\
N(\%) \\
\end{array}$ & $\begin{array}{c}\text { Sim } \\
\mathrm{N}(\%) \\
\end{array}$ & & \\
\hline Gênero & 45 & & & 0,27 & $2,8(0,6-13,0)$ \\
\hline Feminino & & $15(71)$ & $6(29)$ & & \\
\hline Masculino & & $21(88)$ & $3(12)$ & & \\
\hline Estado civil & 45 & & & 0,65 & $1,4(0,2-8,6)$ \\
\hline Sem companheiro & & $30(81)$ & 07 (19) & & \\
\hline Com companheiro & & $6(75)$ & $2(25)$ & & \\
\hline Reside com familiares & 45 & & & 0,67 & $0,6(0,1-2,8)$ \\
\hline Não & & $8(73)$ & $3(27)$ & & \\
\hline Sim & & $28(82)$ & $6(18)$ & & \\
\hline Prática religiosa & 45 & & & 0,46 & $2,2(0,4-12,3)$ \\
\hline Não & & $14(87)$ & $2(13)$ & & \\
\hline Sim & & $22(76)$ & $7(24)$ & & \\
\hline Trabalha & 45 & & & 0,62 & $1,8(0,3-11,1)$ \\
\hline Não & & $31(82)$ & $07(18)$ & & \\
\hline Sim & & $5(71)$ & $2(29)$ & & \\
\hline Renda própria & 45 & & & 0,42 & $2,4(0,5-10,9)$ \\
\hline Não & & $27(84)$ & $5(16)$ & & \\
\hline Sim & & $9(69)$ & $4(31)$ & & \\
\hline $\begin{array}{l}\text { Satisfação com situação } \\
\text { financeira }\end{array}$ & 43 & & & $0,016^{*}$ & $1,5(1,2-1,9)$ \\
\hline Não & & $18(68)$ & $9(33)$ & & \\
\hline Sim & & $16(100)$ & $0(0)$ & & \\
\hline
\end{tabular}

Tabela 3: Caracterização clínica e de prejuízos sociais dos participantes, de acordo com a presença ou ausência de uso problemático de drogas

\begin{tabular}{|c|c|c|c|c|c|}
\hline \multirow{2}{*}{ Variáveis } & \multirow{2}{*}{$\mathrm{N}$ válido } & \multicolumn{2}{|c|}{ Uso problemático de drogas } & \multirow{2}{*}{$\mathrm{p}$} & \multirow{2}{*}{ OR (IC) } \\
\hline & & Não & Sim & & \\
\hline Uso de drogas por familiar & 45 & & & 0,28 & $2,5(0,5-11,6)$ \\
\hline Não & & $16(73)$ & $6(27)$ & & \\
\hline Sim & & $20(87)$ & $3(13)$ & & \\
\hline Dificuldades em usar medicamentos & 45 & & & 0,55 & $1,3(0,2-7,6)$ \\
\hline Não & & $26(79)$ & $7(21)$ & & \\
\hline Sim & & $10(83)$ & $2(17)$ & & \\
\hline Hospitalizações psiquiátricas & 45 & & & 0,35 & $2,5(0,5-12,9)$ \\
\hline Não & & $6(67)$ & $3(33)$ & & \\
\hline Sim & & $30(83)$ & $6(17)$ & & \\
\hline Impulsividade & 44 & & & $0,02^{*}$ & $10,7(1,2-94,7)$ \\
\hline Baixa & & $20(95)$ & $1(5)$ & & \\
\hline Alta & & $15(65)$ & $08(35)$ & & \\
\hline
\end{tabular}


Tabela 3: Continuação

\begin{tabular}{|c|c|c|c|c|c|}
\hline \multirow{2}{*}{ Variáveis } & \multirow{2}{*}{ N válido } & \multicolumn{2}{|c|}{ Uso problemático de drogas } & \multirow{2}{*}{$\mathrm{p}$} & \multirow{2}{*}{ OR (IC) } \\
\hline & & Não & Sim & & \\
\hline Experiência de violência & 45 & & & 0,59 & $1,1(0,3-4,9)$ \\
\hline Não & & $19(79)$ & $5(21)$ & & \\
\hline Sim & & $17(81)$ & $4(19)$ & & \\
\hline Experiência de discriminação & 44 & & & $0,003^{\dagger}$ & $0,1(0,1-0,5)$ \\
\hline Não & & $24(96)$ & $1(04)$ & & \\
\hline Sim & & $11(58)$ & $8(42)$ & & \\
\hline Experiência de viver nas ruas & 45 & & & 0,13 & $0,2(0,0-1,4)$ \\
\hline Não & & $32(84)$ & $6(16)$ & & \\
\hline Sim & & $4(57)$ & $3(43)$ & & \\
\hline Histórico de problemas com a polícia & 45 & & & 0,65 & $1,0(0,2-5,8)$ \\
\hline Não & & $28(80)$ & $7(20)$ & & \\
\hline Sim & & $8(80)$ & $2(20)$ & & \\
\hline
\end{tabular}

${ }^{*} p<0,05 ; \dagger p<0,005$

\section{Discussão}

Os resultados mostraram que as drogas mais prevalentes referidas pelos participantes foram o tabaco, o álcool e a maconha. A maioria dos pacientes $(49 \%)$ referiram consumo problemático de tabaco, em consonância a outros estudos, que mostraram a alta incidência de consumo de tabaco entre pacientes psiquiátricos ${ }^{(25)}$. Esses estudos relacionam as prevalências encontradas ao alívio dos efeitos colaterais dos psicotrópicos ${ }^{(25)}$. Ao considerar que pacientes que referem consumo problemático de drogas geralmente são encaminhados ao CAPS Álcool e Drogas de seu município, o número encontrado de pessoas em tratamento para esquizofrenia e fazendo uso problemático de drogas foi alto, representando $20 \%$ da amostra.

Em relação às variáveis sociodemográficas, a maioria dos participantes não estavam trabalhando no momento da coleta de dados (84\%), não tinham renda própria $(71 \%)$ e estavam insatisfeitos com sua situação financeira (60\%). Essa insatisfação mostrou-se associada ao uso problemático de drogas. Há a hipótese de as pessoas mais insatisfeitas pertencerem a famílias com menores condições econômicas, o que pode ter maiores repercussões com o desemprego. Além disso, os indivíduos com esquizofrenia podem enfrentar dificuldades para desenvolver as atividades laborais, tanto por condições relacionadas à sintomatologia como pelo estigma social e ausência de políticas públicas consolidadas que incentivem a geração de renda, como a economia solidária.

Outro dado importante foi que $46,6 \%$ dos entrevistados já haviam sofrido algum tipo de violência física, sexual, psicológica e/ou contra a propriedade. Apesar de não ter sido encontrada associação entre as situações de violência e o uso problemático de drogas, a literatura afirma que elas acontecem na infância e adolescência, podendo ser elementos que aumentam a suscetibilidade do desdobramento de quadros clínicos psicopatológicos ou o consumo problemático de substâncias psicoativas ${ }^{(26)}$.

Entre os participantes, $43,2 \%$ afirmaram já terem se sentido discriminados por seu quadro clínico, características pessoais ou nível socioeconômico. A percepção de discriminação foi associada ao uso problemático de substâncias. As pessoas com diagnóstico de esquizofrenia ou que fazem uso de substâncias, muitas vezes, sofrem estigmatização e preconceito. Assim, ao relacionar essas duas problemáticas, a estigmatização parece aumentar ${ }^{(27)}$. O serviço de saúde mental é um dos equipamentos de saúde em que o estigma social é mais presente entre os usuários, influenciando no tratamento e na qualidade de vida dos pacientes, direta ou indiretamente ${ }^{(28)}$. O indivíduo com diagnóstico de esquizofrenia e que faz uso de drogas agrega outro estigma ao já existente, de conotação moral, relacionado ao consumo de substâncias ${ }^{(29)}$.

Dentre as variáveis clínicas estudadas, encontrouse associação entre impulsividade e uso problemático de drogas. O comportamento impulsivo ocorre quando o indivíduo toma decisões sem refletir sobre as possíveis consequências. $\mathrm{O}$ uso de drogas parece colaborar com o alívio de alguns sintomas, como os de humor, entretanto, também parece piorar sintomas psicóticos, levando a piores prognósticos. Assim, muitos usuários, mesmo sabendo do alívio causado pela substância, deixam de consumi-la, pois lembram-se dos efeitos ruins que já vivenciaram. Pessoas mais impulsivas teriam mais dificuldades para controlar o comportamento de consumo, focando-se no alívio inicial( ${ }^{(7)}$.

A principal limitação do estudo refere-se à coleta de informações sobre o consumo de drogas por meio do autorrelato, pois sabe-se que essa temática ainda é um assunto difícil de ser conversado. Para minimizar essa 
dificuldade, buscou-se aplicar os instrumentos em sala reservada, apenas na presença de pesquisadores, que eram neutros em relação ao CAPS II. Não foi permitida a presença de familiares ou de profissionais, que poderiam intimidar o usuário.

\section{Considerações Finais}

Este estudo buscou identificar o consumo de substância entre pessoas com esquizofrenia em tratamento em serviços comunitários de saúde mental. Os resultados mostraram a existência do uso problemático de substâncias entre esses pacientes, especialmente, de tabaco, álcool e maconha. Importante notar também que o consumo encontrado faz parte de um contexto mais amplo, que reflete o aumento da autonomia dos pacientes em serviços comunitários. Destaca-se a importância dos profissionais de saúde mental em adotar uma postura aberta para conversar sobre essa temática com seus pacientes, uma postura curiosa para compreender os motivos que levam essas pessoas ao consumo e uma postura colaborativa, para negociar com o usuário estratégias para reduzir ou interromper o consumo ou para reduzir os possíveis danos advindos dele.

A insatisfação com a situação financeira, a experiência de discriminação e a impulsividade foram características que se mostraram associadas ao uso problemático de drogas. No tratamento, é importante abordar as diversas dimensões da vida do indivíduo, pois elas se relacionam com sua saúde. Dificuldades financeiras e situações de estigma e discriminação são importantes gatilhos para o estresse e, consequente, para o adoecimento mental. Treinar habilidades, como o controle de impulsividade, também auxilia a ponderar sobre comportamentos e decisões que impactam na saúde física e mental do indivíduo.

\section{Referências}

1. Arias F, Szerman N, Veja P, Mesias B, Morant C, Ochoa E, Poyo F, Babín F. [Madrid study on the prevalence and characteristics of outpatients with dual pathology in community mental health and substance misuse services]. Adicciones. [Internet]. 2013 [cited 2015 Oct 1]; 25(2):11827. Available from: http://www.patologiadual.es/docs/2EEMARIAS-Dual\%20trastorno12-2-131.pdf Spanish.

2. Bolotner NS, Horcajadas FA, Vich FB, Perez BM, Villamor IB, Morant C, Mangado EO, Calvo FP. [Pilot study on the prevalence of dual pathology in community mental health and substance misuse services in Madrid]. Adicciones. [Internet]. 2011 [cited 2015 Oct 1]; 23(3):249-55. Available from: http:// www.patologiadual.es/docs/1EEM-estudiopiloto.pdf Spanish. 3. Jiménez-Castro L, Raventós-Vorst H, Escamilla, M. [Substance use disorder and schizophrenia: prevalence and sociodemographic characteristics in the Latin American population]. Actas Esp Psiquiatr. [Internet]. 2012 Jul 13 [cited 2015 Oct 1]; 39(2):123-30. Available from: https:// www.ncbi.nlm.nih.gov/pmc/articles/PMC3395996/pdf/ nihms378572.pdf Spanish, English.

4. Regier DA, Farmer ME, Rae DS, Locke BZ, Keith SJ, Judd LL, Goodwin FK. Comorbidity of mental disorders with alcohol and other drug abuse. Results from the Epidemiologic Catchment Area (ECA) Study. JAMA. [Internet]. 1990 Nov 21 [cited 2015 Oct 1]; 264(19): 2511-8. Available from: http://doi.org/10.1001/jama.264.19.2511.

5. Kerner B. Comorbid substance use disorders in schizophrenia: A latent class approach. Psychiatry Res. [Internet]. 2015 Feb 28 [cited 2015 Oct 1]; 225(3):395-401. Available from: http://doi.org/10.1016/j. psychres.2014.12.006.

6. Alves H, Kessler F, Ratto LRC. Comorbidity: alcohol use and other psychiatric disorders. Rev Bras Psiquiatr. [Internet]. 2004 May [cited 2015 Oct 1]; 26(Suppl1):51-3. Available from:http://www.scielo.br/scielo.php?script=sci_ar ttext\&pid=S151644462004000500013\&Ing=en English, Portuguese.

7. Zhornitsky S, Rizkallah E, Pampoulova T, Chiasson JP, Lipp O, Stip E, Potvin S. Sensation-seeking, social anhedonia, and impulsivity in substance use disorder patients with and without schizophrenia and in non-abusing schizophrenia patients. Psychiatry Res. [Internet]. 2012 Dec 20 [cited 2015 Oct 1]; 200(2-3):237-41. Available from: http://doi.org/10.1016/j.psychres.2012.07.046.

8. McCreadie RG. Use of drugs, alcohol and tobacco by people with schizophrenia: case-control study. $\mathrm{Br} \mathrm{J}$ Psychiatry. [Internet]. 2002 Oct [cited 2015 Oct 1]; 181:3215. Available from: http://doi.org/10.1192/bjp.181.4.321.

9. Batki SL, Leontieva L, Dimmock JA, Ploutz-Snyder R. Negative symptoms are associated with less alcohol use, craving, and "high" in alcohol dependent patients with schizophrenia. Schizophr Res. [Internet]. 2008 Oct [cited 2015 Oct 1]; 105(1-3):201-7. Available from: https:// www.ncbi.nlm.nih.gov/pmc/articles/PMC2582942/pdf/ nihms74867.pdf.

10. Lobbana F, Barrowclough C, Jeffery S, Bucci S, Taylor $\mathrm{K}$, Mallinson S, Fitzsimmons M, Marshall M. Understanding factors influencing substance use in people with recent onset psychosis: A qualitative study. Soc Sci Med. [Internet]. 2010 Apr [cited 2015 Oct 1]; 70(8): 1141-7. Available from: http://doi.org/10.1016/j.socscimed.2009.12.026.

11. Huang MC, Yu CH, Chen CT, Chen CC, Shen WW, Chen $\mathrm{CH}$. Prevalence and identification of alcohol use disorders among severe mental illness inpatients in Taiwan. Psychiatry Clin Neurosci. [Internet]. 2009 Jan 15 [cited 2015 Oct 1]; 63(1):94-100. Available from: http://doi. org/10.1111/j.1440-1819.2008.01909.x 
12. Thornton LK, Baker AL, Johnson MP, Kay-Lambkin F, Lewin TJ. Reasons for substance use among people with psychotic disorders: method triangulation approach. Psychol Addict Behav. [Internet]. 2012 Jun [cited 2015 Oct 1]; 26(2):279-88. Available from: http://doi.org/10.1037/ a0026469.

13. Donoghue K, Doody GA, Murray RM, Jones PB, Morgan C, Dazzan P, Hart J, Mazzoncini R, Maccabe JH. Cannabis use, gender and age of onset of schizophrenia: data from the ÆESOP study. Psychiatry Res. [Internet]. 2014 Mar 30 [cited 2015 Oct 1]; 215(3):528-32. Available from: http:// doi.org/10.1016/j.psychres.2013.12.038.

14. Grech A, Takei N, Murray RM. Psychosis and cannabis use. Schizophr Res. [Internet]. 1998 Jan [cited 2015 Oct 1]; 29(1-2):21. Available from: http://doi.org/10.1016/S09209964(97)88341-71998.

15. Grech, A. Should we advise patients with serious mental illness to stop using cannabis? Psychol Med. [Internet]. 2008 Mar [cited 2015 Oct 1]; 38(3):459-64. Available from: http://doi.org/10.1017/S0033291707002255.

16. van Gastel WA, MacCabe JH, Schubart CD, Vreeker A, Tempelaar W, Kahn RS, Boks MP. Cigarette smoking and cannabis use are equally strongly associated with psychotic-like experiences: a cross-sectional study in 1929 young adults. Psychol Med. [Internet]. 2013 Nov [cited 2015 Oct 1]; 43(11):2393-401. Available from: http://doi. org/10.1017/S0033291713000202.

17. Asher CJ, Gask L. Reasons for illicit drug use in people with schizophrenia: Qualitative study. BMC Psychiatry. [Internet]. 2010 Nov 22 [cited 2015 Oct 1]; 10(1):94. Available from: http://doi.org/10.1186/1471-244X-10-94.

18. Costain WF. The effects of cannabis abuse on the symptoms of schizophrenia: Patient perspectives. Int $\mathrm{J}$ Ment Health Nurs. [Internet]. 2008 Aug [cited 2015 Oct 1]; 17(4):227-35. Available from: http://onlinelibrary.wiley.com/ doi/10.1111/j.1447-0349.2008.00538.x/abstract

19. Drake RE, Mueser KT. Co-occurring alcohol use disorder and schizophrenia. Alcohol Res Health. [Internet]. 2002 [cited 2015 Oct 1]; 26(2):99-102. Available from: http://pubs.niaaa.nih.gov/publications/arh26-2/99-102.pdf. 20. Zaleski M, Laranjeira RR, Marques ACPR, Ratto L, Romano M, Alves HNP, Soares MBM Abelardino V, Kessler F, Brasiliano S, Nicastri S, Hochgraf PB, Gigliotti AP, Lemos T. [Guidelines of the Brazilian Association of Studies on Alcohol and Other Drugs (ABEAD) for diagnoses and treatment of psychiatric comorbidity with alcohol and other drugs dependence]. Rev Bras Psiquiatr. [Internet]. 2006 Jun [cited 2015 Oct 1]; 28(2):142-8. Available from: http:// www.scielo.br/pdf/rbp/v28n2/29783.pdf Portuguese.

21. Henrique IFS, de Micheli D, de Lacerda RB, de Lacerda LA, Formigoni MLOS. [Validation of the Brazilian version of Alcohol, Smoking and Substance Involvement Screening Test (ASSIST)]. Rev Assoc Med Bras. [Internet]. 2004
Apr [cited 2015 Oct 1]; 50(2):199-206. Available from: http://dx.doi.org/10.1590/S0104-42302004000200039 Portuguese.

22. WHO ASSIST Working Group. The alcohol, smoking and substance involvement screening test (ASSIST): development, reliability and feasibility. Addiction. [Internet]. 2002 Sep [cited 2015 Oct 1]; 97(9):1183-94. Available from: https://www.ncbi.nlm.nih.gov/pubmed/12199834.

23. Malloy-Diniz LF, Mattos $P$, Leite WB, Neander A, Coutinho G, de Paula JJ, Tavares H, Vasconcelos AG, Fuentes D. [Translation and cultural adaptation of Barratt Impulsiveness Scale (BIS-11) for administration in Brazilian adults]. J Bras Psiquiatr. [Internet]. 2010 [cited 2015 Oct 1]; 59(2): 99-105. Available from: http:// www.scielo.br/scielo.php?script=sci_arttext\&pid=S004720852010000200004\&lng=en Portuguese.

24. Stanford MS, Mathias CW, Dougherty DM, Lake $\mathrm{SL}$, Anderson NE, Patton JH. Fifty years of the Barrat Impulsiveness Scale: an update and review. Pers Individ. [Internet]. 2009 Oct [cited 2015 Oct 1]; 47(5): 385-95. Available from: http://dx.doi.org/10.1016/j.paid.2009.04.008 25. Pettersen H, Ruud T, Ravndal E, Landheim A. Walking the fine line: Self-reported reasons for substance use in persons with severe mental illness. Int J Qual Stud Health Well-being. [Internet]. 2013 [cited 2015 Oct 1]; 1(8):1-11. Available from: http://www.ijqhw.net/index.php/qhw/article/ view/21968.

26. Huang MC, Schwandt ML, Ramchandani VA, George DT, Heilig M. Impact of multiple types of childhood trauma exposure on risk of psychiatric comorbidity among alcoholic inpatients. Alcohol Clin Exp Res. [Internet]. 2012 Jun [cited 2015 Oct 1]; 36(6):1099-107. Available from: http://doi. org/10.1111/j.1530-0277.2011.01695.x

27. Rüsch N, Angermeyer MC, Corrigan PW. Mental illness stigma: concepts, consequences, and initiatives to reduce stigma. Eur Psychiatry. [Internet]. 2005 Dec [cited 2015 Oct 1]; 20(8):529-39. Available from: http://doi.org/10.1016/j. eurpsy.2005.04.004.

28. Neves DP. Alcoholism: indictment or diagnosis?. Cad Saúde Pública. [Internet]. 2004 Feb [cited 2015 Oct 1]; 20(1):7-14. Available from: http://www.scielo.br/pdf/csp/ v20n1/02.pdf Portuguese.

29. Rondina RC, Gorayeb R, Botelho C. [Relationship between smoking behavior and psychiatric disorders]. Rev Psiq Clin. [Internet]. 2003 Jun 30 [cited 2015 Oct 1]; 30(6), 221-8. Available from: http://www.scielo.br/pdf/rpc/v30n6/ a05v30n6.pdf Portuguese. 